\title{
Reactive transport benchmarks for subsurface environmental simulation
}

\author{
Carl I. Steefel $^{1}$ - Steven B. Yabusaki ${ }^{2}$ K. Ulrich Mayer ${ }^{3}$
}

Published online: 5 June 2015

(C) Springer International Publishing Switzerland 2015

\section{Objectives of the special issue}

Over the last 20 years, we have seen firsthand the evolution of multicomponent reactive transport modeling and the expanding range and increasing complexity of subsurface applications it is being used to address. There is a growing reliance on reactive transport modeling (RTM) to address some of the most compelling issues facing our planet: climate change, nuclear waste management, contaminant remediation, and pollution prevention. While these issues are motivating the development of new and improved capabilities for subsurface environmental modeling using RTM (e.g., biogeochemistry from cell-scale physiology to continental-scale terrestrial ecosystems, nonisothermal multiphase conditions, coupled geomechanics), there remain longstanding challenges in characterizing the natural variability of hydrological, biological, and geochemical properties in subsurface environments and limited success in transferring models between sites and across scales.

An equally important trend over the last 20 years is the evolution of modeling from a service sought out after data has been collected to a multifaceted research approach that

Carl I. Steefel

CISteefel@lbl.gov

1 Earth Sciences Division,

Lawrence Berkeley National Laboratory

Berkeley, CA 94720 USA

2 Pacific Northwest National Laboratory

Richland, WA 99352 USA

3 Department of Earth, Ocean, and Atmospheric Sciences, University of British Columbia Vancouver, BC, Canada provides (1) an organizing principle for characterization and monitoring activities; (2) a systematic framework for identifying knowledge gaps, developing and integrating new knowledge; and (3) a mechanistic understanding that represents the collective wisdom of the participating scientists and engineers [1]. There are now large multidisciplinary projects where the research approach is model-driven, and the principal product is a holistic predictive simulation capability that can be used as a test bed for alternative conceptualizations of processes, properties, and conditions [2, 3]; http://today.lbl.gov/2013/08/22/project-explores-linksbetween-subsurface-genomics-watershed-biogeochemistry/; http://newscenter.lbl.gov/2015/04/01/ngee-tropics/.

Much of the future growth and expanded role for RTM will depend on its continued ability to exploit technological advancements in the earth and environmental sciences. Advances in measurement technology, particularly in molecular biology (genomics), isotope fractionation, and high-resolution X-ray spectroscopy, have created new lines of research that can be used to inform the conceptualization of reactions and rate laws and validate mechanistic models. For example, spectroscopy has identified the oxidation states of key components and elemental distributions at increasingly smaller scales and lower concentrations; molecular biology has progressed from identifying the presence of microbes to characterization of which microbial communities are active and what they are doing (i.e., microbial function), which has led in turn to the identification of active processes under conditions beyond what analytical chemistry can discern; isotope ratios in pore water and solid phases that can be used to distinguish between biotic from abiotic processes, sorption from precipitation, and origin and age of groundwater.

The other noteworthy development that is expanding the role of RTM in subsurface environmental modeling is 
the advance in computational technology that is enabling the simulation of more coupled processes with increasing mechanistic detail. In some cases, this involves the inclusion of more reactive species and/or microbial populations in the simulations; in other cases, the impact is through the ability to achieve high resolution of property distributions over longer simulated times.

To achieve these ambitious objectives for subsurface reactive transport simulation, the subsurface science and engineering community is being driven to provide accurate assessments of engineering performance and risk for important issues with far-reaching consequences. As a result, the complexity and detail of subsurface processes, properties, and conditions that can be simulated have significantly expanded. This expansion was enabled, in part, by advances in measurement technology, computing technology, and numerical techniques. The ever-increasing levels of sophistication bring also greater opportunities for error, so how does one establish the validity of a simulator formulation prior to application? Closed form solutions are necessary and useful, but limited to situations that are far simpler than typical applications that combine many physical and chemical processes, in many cases in coupled form. In the absence of closed form and yet realistic solutions for complex applications, benchmark problems with an accepted set of results will be indispensable to qualifying codes for various environmental applications.

The intent of this special issue is to develop and publish a set of well-described benchmark problems that can be used to demonstrate simulator conformance with norms established by the subsurface science and engineering community. In every case, the primary focus in the manuscripts is on the development of a benchmark that provides rigorous tests of one or more essential features of a numerical reactive transport simulator. The objective is not to verify this or that specific code-the reactive transport codes play a supporting role in this regard-but rather to use the codes to verify that a common solution of the problem can be achieved. Each benchmark was required to have a minimum of three different codes to obtain the same or nearly the same results, thus reducing the likelihood that the benchmark results used to verify future codes are inaccurate for the particular benchmark considered.

Thus, the objective of each of the manuscripts is to present an environmentally relevant benchmark problem that tests the conceptual model capabilities, numerical implementation, process coupling, and accuracy. Each benchmark contribution consists of a principal comprehensive problem and supporting component problems. This is intended to isolate key attributes of the benchmark problem for higher scrutiny before addressing the full complement of interacting processes, properties, and conditions. The intent is to provide an improved basis for resolving discrepancies between code bases, but this feature should also increase the number of problems that can be attempted by interested modelers. More complex problems generally follow the base case problems.

The manuscripts collected in this special issue in fact are the outgrowth of four workshops held on the topic over the course of 3 years. The first Subsurface Environmental Simulation workshop was held in Berkeley, CA on November 16-18, 2011 and was attended by about 45 participants (C.I. Steefel, organizer). The second workshop was held in Zhongli Taiwan, on October 29-31, 2012 (G.T. Yeh, organizer). The third workshop was held on November 11-13, 2013 at the Helmholtz Centre for Environmental Research in Leipzig, Germany (O. Kolditz, organizer). The last of the workshops was held in Cadarache, France, on October 6-8, 2014 (O. Bildstein, organizer).

\section{Intended audience}

The primary audience for this special issue is of course the model developers who will benefit from the availability of a set of rigorous benchmarks for complex subsurface environmental problems. Anybody who has been involved in code development, whether specifically for RTM or for some other application(s) in the earth and environmental sciences, will appreciate the value of a reliable set of benchmarks to test their own code against. This can dramatically shorten the development time for complex environmental software. The focus in this special issue has been on establishing the accuracy of complex simulations rather than on computational performance. This second objective could be pursued relatively easily by taking the codes summarized in this issue [4], or other codes as feasible, and comparing their performance on the benchmark problems using the same space and time discretization.

The second audience for the benchmarks published here are the model users who are interested in exploring a range of environmental applications that can be addressed by reactive transport modeling. With that in mind, these benchmark problems are actually instructional, providing a progression from conceptualization to the specification of an actual reaction network. Even for experienced modelers, these benchmarks can provide an entrée into new reaction processes and provide templates that can be adapted for other specifications. For example, one can see how isotopic techniques can be incorporated into reactive transport simulators. Others can learn how the range of complexity in mineral surfaces can be addressed by multi-site and multi-rate models of uranium surface complexation. In this respect, we hope the special issue aids in the training of a new generation of modelers who continue to expand the role of reactive transport modeling in subsurface environmental simulation. 


\section{Contents of the special issue}

The special issue begins with a summary of the capabilities of the modern numerically based reactive transport codes that are suitable for subsurface environmental simulation. All of the codes represented here are based on a continuum approach. A high-level and non-exhaustive overview of the mathematical and numerical bases of the modern reactive simulators is provided in the first part of the paper (Reactive transport benchmarks for subsurface environmental simulation; [4]). Some of the RTM simulators do not follow precisely the formulations presented there, but we think the summary provided here is useful, particularly since there is interest in the RTM community in avoiding strictly "black box" approaches to modeling (one expects that the model users will at least have a good idea of what system of equations are being solved). The second part of this overview paper focuses on short summaries of many of the commonly available RTM simulators, although again the list here is not exhaustive. Some codes were not included simply because none of the benchmark workshop participants and/or authors offered to apply them to the problems. Others were considered in the less than mature stage and thus not suitable for benchmarking at this point in time.

The benchmark problems in the remainder of the special issue are organized by topic, including (1) microbially mediated reactions, (2) isotopes, (3) multi-component diffusion, (4) uranium fate and transport, (5) metal mobility in mining affected systems, and (6) waste repositories and related aspects.

\subsection{Microbially mediated reactions}

In a benchmark focusing on modeling microbially mediated reactions (A benchmark for microbially mediated chromium reduction under denitrifying conditions in a biostimulation column experiment [5]), Cr reduction in a 1D column is investigated with standard Monod (or Michaelis-Menten) rate formulations. The benchmark represents a simplification of an actual column experiment [6] that uses sediment and chemical conditions from a contaminated site at the Hanford Nuclear Reservation in Washington, USA. The remarkable agreement between the codes on this benchmark likely reflects in part the focus on kinetic as opposed to thermodynamic formulations, which proved to be the biggest source of discrepancies in many of the benchmarks.

\subsection{Isotopes}

A benchmark problem on isotopic fractionation consists of four problem levels and was developed for the simulation of kinetic $\mathrm{Cr}$ isotope fractionation in 1D and 2D domains (Benchmarking the simulation of $\mathrm{Cr}$ isotope fractionation [7]). The benchmark is based on a recent field study where $\mathrm{Cr}(\mathrm{VI})$ reduction and accompanying $\mathrm{Cr}$ isotope fractionation occurs abiotically through reaction with dissolved Fe(II) [8]. The benchmark demonstrates that excellent agreement can be obtained between codes, despite differences in the approaches to implement isotope fractionation.

\subsection{Multi-component diffusion}

Two benchmarks treat the problem of multi-component diffusion, which may be a particularly important effect where low hydraulic conductivity materials are present within the subsurface. While these diffusion-dominant zones may be mostly bypassed by the principal groundwater flow, they can be important where contamination is located within them. They are also important in geological and engineered waste repositories where low conductivity, clay-rich zones provide part of the safety case. The first benchmark investigates the role of multi-component diffusion based on the Nernst-Planck equation rather than the more common (and simpler) treatment based on Fick's law (Benchmarks for multicomponent diffusion and electrochemical migration [9]). The behavior observed in the benchmark simulations arises from the effects of electrochemical migration that occurs when charged species diffuse at different rates. A second benchmark (Benchmark reactive transport simulations of a column experiment [10]) simulates multi-species diffusion (Nernst-Planck equation) through compacted bentonite and includes also an explicit treatment of electrostatic effects associated with charged clay surfaces. Bentonite is an important component of the engineered barrier system for many nuclear waste repositories.

\subsection{Uranium fate and transport}

Uranium mobility is notoriously sensitive to $\mathrm{pH}, \mathrm{Eh}$, alkalinity, major ions, surface sites, etc. The three benchmarks that consider uranium fate and transport represent some of the successes in using coupled process modeling to develop a systematic understanding of uranium behavior in naturally complex subsurface materials. The first benchmark is based on a field experiment conducted at the Rifle site in western CO, USA (A uranium bioremediation reactive transport benchmark [11]) in which acetate was injected into uranium-contaminated groundwater to drive microbially mediated reduction and immobilization of uranium. The problem is simplified somewhat in terms of the hydrogeology of the site, but includes a complex biogeochemical reaction network affecting the reduction and sorption of the uranium. A second benchmark focuses on the important role of Fe-hydroxides in re-oxidizing biogenically reduced uranium (A reactive transport benchmark on modeling 
biogenic uraninite re-oxidation by $\mathrm{Fe}(\mathrm{III})$-(hydr)oxides [12]). A third benchmark focuses on multi-rate models used to simulate uranium leaching and migration (A benchmark for multi-rate surface complexation and 1-D dual domain multicomponent reactive transport of U(VI) [13]).

\subsection{Metal mobility in mining affected areas}

Two benchmarks consider metal mobility in the context of mining activities. The first (Benchmark problems for reactive transport modeling of the generation and attenuation of acid rock drainage [14]) uses reactive transport modeling to simulate the generation of acidity as a result of sulfide oxidation and its subsequent effect on metal mobility above and below the water table. The second benchmark focuses on metal accumulation and mobility in lake sediments downstream of mining operations (A reactive transport benchmark on heavy metal cycling in lake sediments [15]). The modeling assumes a 1D geometry that extends from the lake-sediment interface to depth and includes component problems with and without sediment burial.

\subsection{Waste repositories and related aspects}

Three benchmarks focus on topics related to the storage of nuclear waste in geological repositories. The first addresses reactive transport associated with a cement-clay interface as might be expected in the engineered barrier system for a waste repository (Benchmark for multicomponent reactive transport codes across a cement/clay interface [16]). The challenges in this problem result in part from the high geochemical contrast between the cement and clay, which drives diffusive mixing across the sharp interface. The large concentration gradients in the context of the nonlinear mineral dissolution and precipitation provide an excellent test for the robustness of the simulators. The second benchmark relevant to nuclear waste repositories considers the effects of porosity, permeability and tortuosity evolution in 1D and 2D systems (Implementation and evaluation of permeability-porosity and tortuosity-porosity relationships linked to mineral dissolution-precipitation [17]). The porosity reduction is the result of reactions (principally gypsum precipitation), which in turn decrease the permeability to the point where flow is effectively stopped. In the 2D case, the same set of reactions affects porosity and permeability in a heterogeneous domain. The last benchmark focuses on simulations of reactive transport and solute mobility resulting from cracks developed in concrete (Decalcification of cracked cement structures [18]). The problem shares some similarities with the Marty et al. [16] benchmark described above, but also includes the effect of discrete fractures on the system behavior.
Acknowledgments The preparation of the special issue and the workshops were supported in part by the Genomes to Watershed Science Focus Area at Lawrence Berkeley National Laboratory funded by the U.S. Department of Energy, Office of Science, Biological and Environmental Research under Contract No. DE-AC02-05CH11231 (CIS, SBY). Additional funding was provided by the Director, Office of Science, Office of Basic Energy Sciences, Chemical Sciences, Geosciences, and Biosciences Division of the U.S. Department of Energy under Contract No. DE-AC02-05CH11231 (CIS).

\section{References}

1. Steefel, C.I., DePaolo, D., Lichtner, P.C.: Reactive transport modeling: an essential tool and a new research approach for the earth sciences. Earth Planet. Sci. Lett. 240, 539-558 (2005)

2. Davis, J.A., Yabusaki, S.B., Steefel, C.I., Zachara, J.M., Curtis, G.P., Redden, G.P., Criscenti, L.J., Honeyman, B.D.: Assessing conceptual models for subsurface reactive transport of inorganic contaminants. EOS 85(44), 449 (2004)

3. Steefel, C.I.: The GEWaSC framework: multiscale modeling of coupled biogeochemical, microbiological, and hydrological processes, 2014 Goldschmidt Conference, Sacramento, CA, June 13 (2014)

4. Steefel, C.I., Appelo, C.A.J., Arora, B., Jacques, D., Kalbacher, T., Kolditz, O., Lagneau, V., Lichtner, P.C., Mayer, K.U., Meeussen, J.C.L., Molins, S., Moulton, D., Shao, H., Simůnek, J., Spycher, N., Yabusaki, S.B., Yeh, G.T.: Reactive transport codes for subsurface environmental simulation. Comput. Geosci. (2015). doi:10.1007/s10596-014-9443-x

5. Molins, S., Greskowiak, J., Wanner, C., Mayer, K.U.: A benchmark for microbially mediated chromium reduction under denitrifying conditions in a biostimulation column experiment. Comput. Geosci. (2015). doi:10.1007/s10596-014-9432-0

6. Beller, H.R., Yang, L., Varadharajan, C., Han, R., Lim, H.C., Karaoz, U., Molins, S., Marcus, M.A., Brodie, E.L., Steefel, C.I., Nico, P.: 2014 Divergent aquifer biogeochemical systems converge on similar and unexpected $\mathrm{Cr}(\mathrm{VI})$ reduction products. IEnviron. Sci. Technol. (2014). doi:10.1021/es5016982

7. Wanner, C., Druhan, J.L., Amos, R.T., Alt-Epping, P., Steefel, C.I.: Benchmarking the simulation of $\mathrm{Cr}$ isotope fractionation. Comput. Geol. (2015). doi:10.1007/s10596-014-9436-9

8. Wanner, C., Zink, S., Eggenberger, U., Maeder, U.: Assessing the $\mathrm{Cr}(\mathrm{VI})$ reduction efficiency of a permeable reactive barrier using $\mathrm{Cr}$ isotope measurements and $2 \mathrm{D}$ reactive transport modeling. $\mathrm{J}$. Contam. Hydrol. (2012). doi:10.1015/j.conhydr.2012.01.007

9. Rasouli, P., Steefel, C.I., Mayer, K.U., Rolle, M.: Benchmarks for multicomponent diffusion and electrochemical migration. Comput. Geol. (2015). doi:10.1007/s10596-015-9481-z

10. Alt-Epping, P., Tournassat, C., Rasouli, P., Steefel, C.I., Mayer, K.U., Jenni, A., Mäder, U., Sengor, S., Fernandez, R.: Benchmark reactive transport simulations of a column experiment in compacted bentonite with multi-species diffusion and explicit treatment of electrostatic effects. Comput. Geosci. (2015). doi:10.1007/s10596-014-9451-x

11. Yabusaki, S.B., Şengör, S.S., Fang, U.: A uranium bioremediation reactive transport benchmark. Comput. Geosci. (2015). doi:10.1007/s10596-015-9474-y

12. Şengör, S.S., Mayer, K.U., Greskowiak, J., Wanner, C., Su, D., Prommer, H.: A reactive transport benchmark on modeling biogenic uraninite re-oxidation by $\mathrm{Fe}(\mathrm{III})$-(hydr)oxides. Comput. Geosci. (2015). doi:10.1007/s10596-015-9480-0

13. Greskowiak, J., Gwo, J., Jacques, D., Yin, J., Mayer, K.U.: A benchmark for multi-rate surface complexation and 1-D dual 
domain multicomponent reactive transport of U(VI). Comput. Geosci. (2015). doi:10.1007/s10596-014-9457-4

14. Mayer, K.U., Alt-Epping, P., Jacques, D., Arora, B., Steefel, C.I.: Benchmark problems for reactive transport modeling of the generation and attenuation of acid rock drainage. Comput. Geosci. (2015). doi:10.1007/s10596-015-9476-9

15. Arora, B., Şengör, S.S., Spycher, N., Steefel, C.I.: A reactive transport benchmark on heavy metal cycling in lake sediments. Comput. Geosci. (2015). doi:10.1007/s10596-014-9445-8

16. Marty, N.C.M., Blanc, P., Bildstein, O., Claret, F., Cochepin, B., Danyang, S., Gaucher, E., Jacques, D., Lartigue, J.-E., Mayer, K.U., Meeussen, J.C.L., Munier, I., Pointeau, I., Sanheng, L.,
Steefel, C.I.: Benchmarks for multicomponent reactive transport across a cement/clay interface. Comput. Geosci. (2015). doi:10.1007/s10596-014-9463-6

17. Xie, M., Mayer, K.U., Claret, F., Alt-Epping, P., Diederik, J., Steefel, C.I., Chiaberge, C., Simunek, J.: Implementation and evaluation of permeability-porosity and tortuosityporosity relationships linked to mineral dissolution-precipitation (2015). doi:10.1007/s10596-014-9458-3

18. Perko, J., Mayer, K.U., Kosakowski, G., De Windt, L., Govaerts, J., Jacques, D., Su, D., Meeussen, J.C.L.: Decalcification of cracked cement structures. Comput. Geosci. (2015). doi:10.1007/s10596-014-9467-2 